\title{
Downregulated Th17 responses are associated with reduced gastritis in Helicobacter pylori-infected children
}

\author{
C Serrano $^{1,8}$, SW Wright ${ }^{2,8}$, D Bimczok ${ }^{2}$, CL Shaffer ${ }^{3,4}$, TL Cover ${ }^{3,4}$, A Venegas $^{5}$, MG Salazar ${ }^{6}$, \\ LE Smythies ${ }^{2}$, PR Harris ${ }^{1}$ and PD Smith ${ }^{2,7}$
}

Helicobacter pylori induces less gastric inflammation in children than adults. Here we investigated whether this reduced inflammation involves dysregulated T helper type 17 (Th17) responses. $H$. pylori-infected children and adults in Santiago, Chile had similar levels of $H$. pylori colonization, proportions of bacteria containing cag $A$ and $s 1 / s 2$ vac $A$ markers of virulence, and strain genotypes (predominantly hpEurope), but the children had significantly reduced levels of gastric inflammation and neutrophil infiltration. The reduced neutrophil accumulation in the infected children was accompanied by significantly fewer gastric Th17 cells and significantly lower levels of interleukin (IL)-17-specific mRNA and protein compared with the infected adults. The gastric mucosa of $H$. pylori-infected children also contained higher numbers of IL- $10^{+}$cells and increased levels of both IL-10 and Foxp3 mRNA compared with that of the infected adults. Thus, reduced gastric inflammation, including diminished neutrophil accumulation, in $H$. pylori-infected children compared with infected adults is likely due to downregulated gastric Th17/IL-17 responses as a consequence of enhanced mucosal regulatory T-cell activity in the children.

\section{INTRODUCTION}

The inflammation associated with Helicobacter pylori infection is mediated by gastric mucosal $\mathrm{T}$ helper type 1 (Th1) cells through the release of interferon (IFN)- $\gamma$, based on studies in animal models ${ }^{1-3}$ and adult humans. ${ }^{4-8}$ Study of the infection in adult subjects addresses late $H$. pylori-induced mucosal responses, whereas the study of $H$. pylori infection in children, which has received little investigative attention, offers the opportunity to investigate early mucosal responses to the bacteria. To begin to elucidate the early, possibly age-related, mucosal events in H. pylori infection, we $\mathrm{e}^{8,9}$ and others ${ }^{10}$ have shown that childhood $H$. pylori infection is associated with significantly reduced levels of gastric inflammation and ulceration compared with adults. We also have shown that the gastric mucosa of $H$. pylori-infected children contains increased numbers of regulatory $\mathrm{T}$ (Treg) cells compared with the mucosa of infected adults, ${ }^{8}$ suggesting Treg participation in the reduced Th1-mediated gastritis and ulceration in the children. In this connection, emerging evidence suggests that Treg-mediated immune regulation in humans may contribute to $H$. pylori persistence ${ }^{11,12}$ and that inadequate or absent Treg responses in adult humans and mice are associated with increased mucosal inflammation during $H$. pylori infection. $^{12-15}$

Recent studies also have shown that the levels of interleukin (IL)-17 are increased in the gastric mucosa of mice infected with H. pylori, ${ }^{16-18}$ suggesting that Th17 cells participate in H. pylori gastritis, at least in mice. Indeed, IL-17 is one of the earliest cytokines detected in the gastric mucosa of $\mathrm{H}$. pylori-infected mice. ${ }^{17}$ IL-17 mediates the recruitment and activation of polymorphonuclear neutrophils, a key cellular element in the inflammatory lesion associated with $\mathrm{H}$. pylori infection. ${ }^{19}$ Here

\footnotetext{
${ }^{1}$ Division of Pediatrics, Unit of Gastroenterology and Nutrition, School of Medicine, Universidad Catolica de Chile, Santiago, Chile. ${ }^{2}$ Division of Gastroenterology and Hepatology, University of Alabama at Birmingham, Birmingham, Alabama, USA. ${ }^{3}$ Division of Infectious Diseases, Department of Medicine, Vanderbilt University, Nashville, Tennessee, USA. ${ }^{4}$ Veterans Affairs Tennessee Valley Healthcare System, Nashville, Tennessee, USA. ${ }^{5}$ Department of Molecular Genetics and Microbiology, Faculty of Biological Sciences, Pontificia, Universidad Catolica de Chile, Santiago, Chile. ${ }^{6}$ Division of Hematology/Oncology, Department of Medicine, University of Alabama at Birmingham, Birmingham, Alabama, USA. ${ }^{7}$ Veterans Affairs Medical Center, Birmingham, Alabama, USA and ${ }^{8}$ These authors contributed equally to this work. Correspondence: PD Smith (pdsmith@uab.edu)
} 
we extend our study of the immunopathogenesis of H. pylori gastritis in children as a window into the early cellular responses to the infection by investigating gastric Th17 responses in the H. pylori-infected children and adults living in Chile, where H. pylori is endemic.

\section{RESULTS}

\section{Reduced levels of gastric inflammation in $H$. pylori-infected children compared with infected adults}

To elucidate the immune mechanisms that initiate inflammation in early $H$. pylori infection, we first evaluated gastric tissue specimens from children and adults with or without $H$. pylori infection for inflammation. H. pylori infection was associated with gastric inflammation in both children and adults compared with their non-infected peers (each $P=0.0002$; Figure 1a). However, the mean level of inflammation in the infected children was significantly less compared with that of the infected adults $(P=0.002)$, based on histological evaluation of coded biopsy specimens using the modified Sydney classification. The same biopsy specimens were evaluated for $H$. pylori colonization. The mean level of $H$. pylori colonization in the gastric mucosa of the children and adults was nearly identical (Figure 1b), despite the reduced level of gastritis in the children. Importantly, at each level of bacterial colonization, the $H$. pylori-infected children displayed a reduced histology score compared with that of the infected adults $(P=0.018$; Figure 1c), indicating that the reduced mean histology score in the children (Figure 1a) was not the consequence of a disproportionally lower amount of
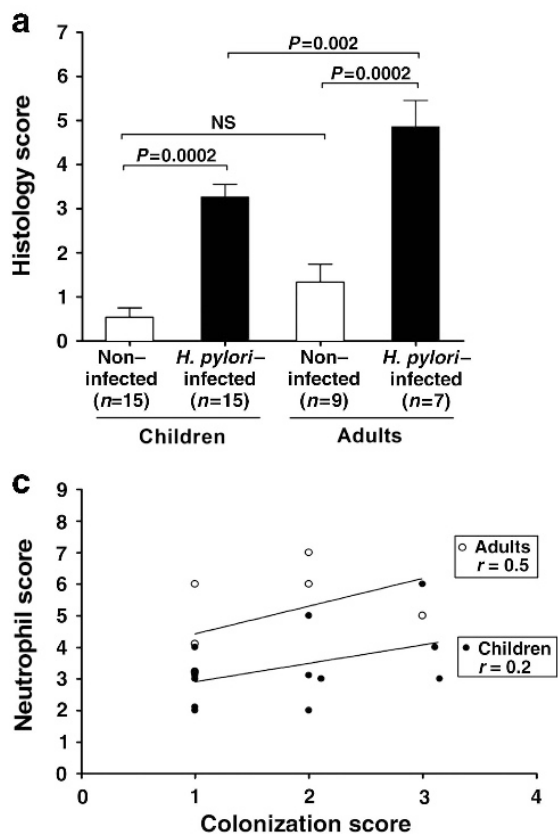

inflammation in the children or an excessively higher amount of inflammation in the adults for any single level of colonization. Rather, $\mathrm{H}$. pylori induced a proportionally lower inflammatory response in children compared with adults at each level of colonization. In addition, the amount of neutrophil infiltration into the gastric mucosa, based on the neutrophil score, was reduced at each level of colonization $(P=0.01$; Figure $1 \mathbf{d})$.

\section{H. pylori from infected children and adults display similar genetic features}

To determine whether the reduced inflammation in the H. pylori-infected children could reflect infection by less virulent bacteria, we characterized the $H$. pylori isolated from 12 of the 15 infected children and all 7 of the infected adults for the presence of $c a g A$ and $v a c A$ alleles and for phylogeographic origin. Among these isolates, the proportion of bacteria that carried cagA and vacA (type s1/s2) markers of virulence was similar in the infected children and adults $(P=0.2$ and 0.1 , respectively; Table 1). All of the strains containing s1 forms of $v a c A$ were classified as subtype s1b. H. pylori isolated from the children and adults also were subjected to nucleotide sequence analysis of the urel, muty, efp, $p p a, a t p A$, and $\operatorname{trp} C$ loci and then compared with 331 reference strains to assess phylogenetic similarity. As shown in the phylogenetic tree (Figure 2), all 12 of the isolates from children and 5 of the 7 isolates from adults were of European origin, ${ }^{20,21}$ suggesting that hpEurope is the predominant $H$. pylori genotype present in this region of Chile. Thus, the Chilean children and adults in our study were colonized by $H$. pylori with similar cagA and vacA profiles and
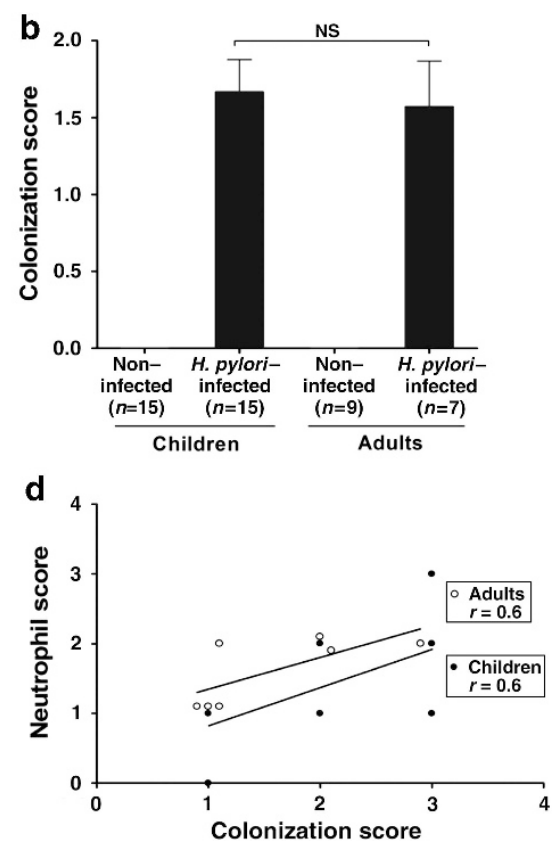

Figure 1 Levels of gastric inflammation and Helicobacter pylori colonization in children and adults. (a) $H$. pylori induced inflammation in the gastric mucosa of both children and adults, but inflammation was significantly less severe in the infected children. (b) Levels of $H$. pylori colonization in gastric mucosa were similar in the infected children and adults. Levels of gastric inflammation and colonization are presented as the histology score and colonization score (mean \pm s.e.m.), respectively (see Methods). H. pylori-infected children had reduced levels of (c) gastric inflammation based on the histology score and (d) neutrophil infiltration based on the neutrophil score compared with the infected adults. (for comparison of lines: in $\mathbf{c}, P=0.018$ and in $\mathbf{d}, P=0.01)$. NS, not significant. 
predominantly of the hpEurope genotype, indicating that host, not bacterial, factors contribute to the reduced gastritis in children.

\section{Reduced frequency of Th17 cells and IL-17 response in the gastric mucosa of $\boldsymbol{H}$. pylori-infected children compared with adults}

As H. pylori gastritis is mediated by $\mathrm{Th} 1^{1-8}$ and possibly Th1 $17^{16-18,22}$ pathway responses, and as the $H$. pylori-infected children display a reduced gastric Th1 response, ${ }^{8}$ we next investigated whether the infected children also have a reduced gastric Th17 response. To determine the prevalence of Th17

Table 1 Helicobacter pylori virulence factors

\begin{tabular}{lccc}
\hline & \multicolumn{2}{c}{ Source of $\boldsymbol{H}$. pylori } & \\
\cline { 2 - 3 } & Children $(\boldsymbol{n}=\mathbf{1 2})$ & Adults $(\boldsymbol{n}=\mathbf{7})$ & P-value \\
\hline CagA, $n(\%)$ & $11(92)$ & $5(71)$ & 0.2 \\
VacA S alleles & & & 0.1 \\
s1 allele, $n(\%)$ & $4(33)$ & $5(71)$ & \\
s2 allele, $n(\%)$ & $8(66)$ & $2(29)$ & \\
\hline
\end{tabular}

cells in gastric mucosa, we used immunofluorescence to analyze gastric biopsies from the $H$. pylori-infected children and adults for $\mathrm{CD}^{+} \mathrm{T}$ cells that expressed IL-17 (Figure 3a). IL-17 expression was detected predominantly in $\mathrm{CD}^{+}{ }^{+}$cells in the lamina propria and occasionally in $\mathrm{CD}^{-}$cells in the epithelium and lamina propria (not shown). The proportion of $\mathrm{CD}^{+} \mathrm{T}$ cells that expressed IL-17 in the gastric lamina propria of $H$. pylori-infected adults was significantly increased compared with that of both the noninfected adults $(P=0.008)$ and infected children $(P=0.024$; Figure $3 \mathbf{b})$. By contrast, the proportion of gastric $\mathrm{IL}-17^{+} /$ $\mathrm{CD}^{+} \mathrm{T}$ cells in the infected children was not significantly increased compared with that of the non-infected children (Figure 3b). Thus, the number of Th17 cells in the gastric mucosa of $H$. pylori-infected children is significantly lower than that of the infected adults.

Because Th17-derived IL-17 drives the recruitment and activation of neutrophils in the intestinal mucosa, we next measured the IL-17 response in gastric tissue specimens from the children and adults. $H$. pylori infection in the children did not induce increased gastric IL-17 mRNA expression, whereas infection in the adults was associated with a several fold increase in IL-17 mRNA ( $P=0.03$; Figure 4a). Consistent with

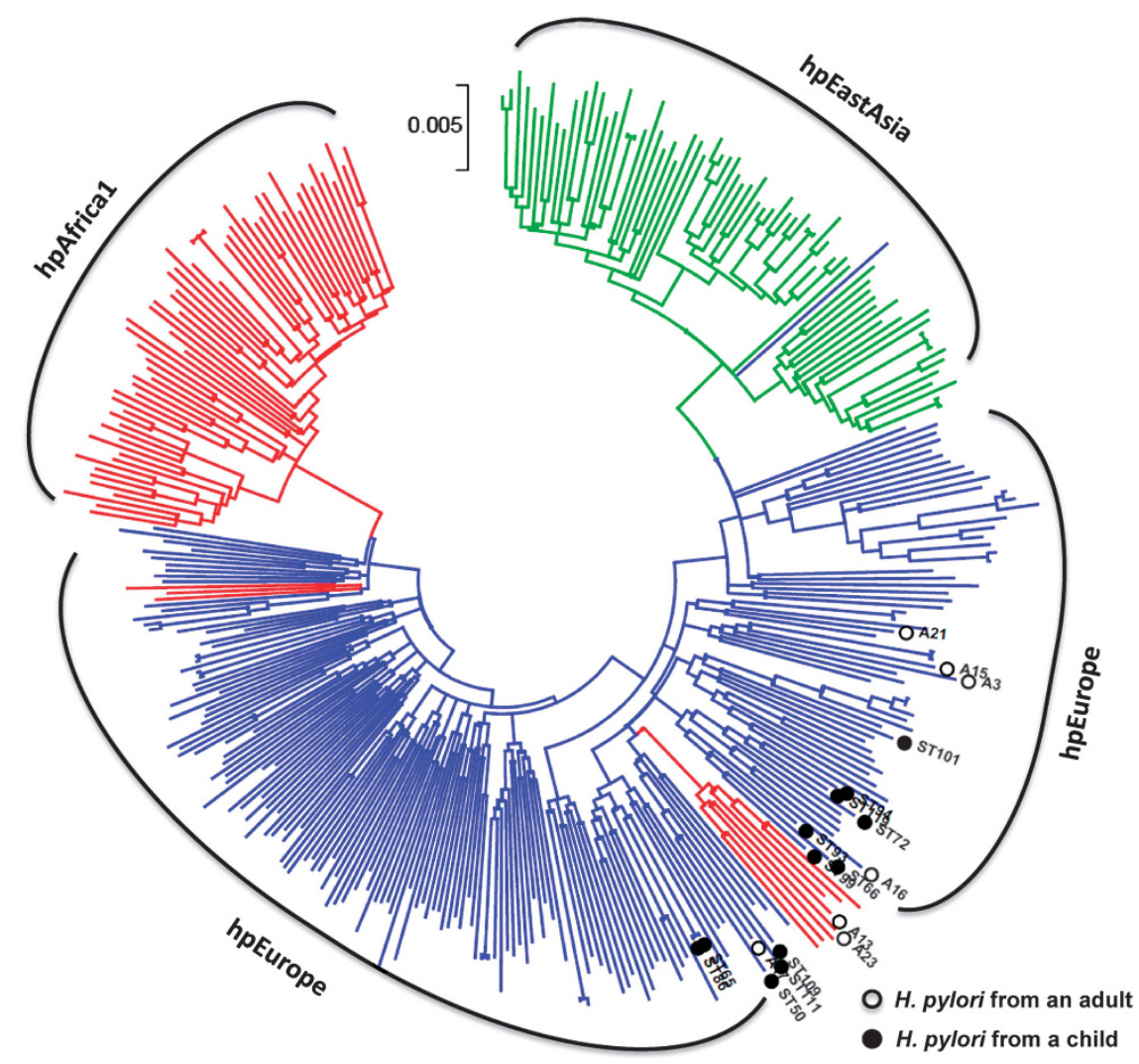

Figure 2 Phylogeographic origin of Helicobacter pyloristrains. H. pylori isolated from the children ( $n=12$, open circles) and adults ( $n=7$, closed circles) were subjected to nucleotide sequence analysis of the urel, muty, efp, ppa, atpA, and trpCloci. Concatenated nucleotide sequences of the loci from the 19 isolates were aligned to the corresponding sequences of 331 reference strains before phylogenetic analysis. ${ }^{20,50}$ The phylogenetic tree is drawn to scale with branch lengths in the same units as those of the evolutionary distances used to infer the tree. Major strain populations are designated as hpEurope (blue), hpAfrica1 (red), and hpEastAsia (green) (including subgroups hspEAsia (green) and hspAmerind (green)). Seventeen of 19 H. pylori isolates were hpEurope and 2 were hpAfrica. 

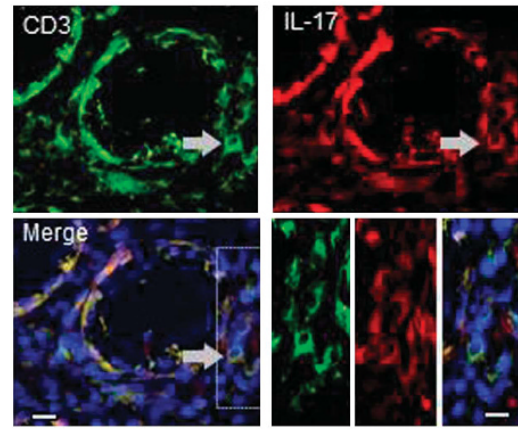

b

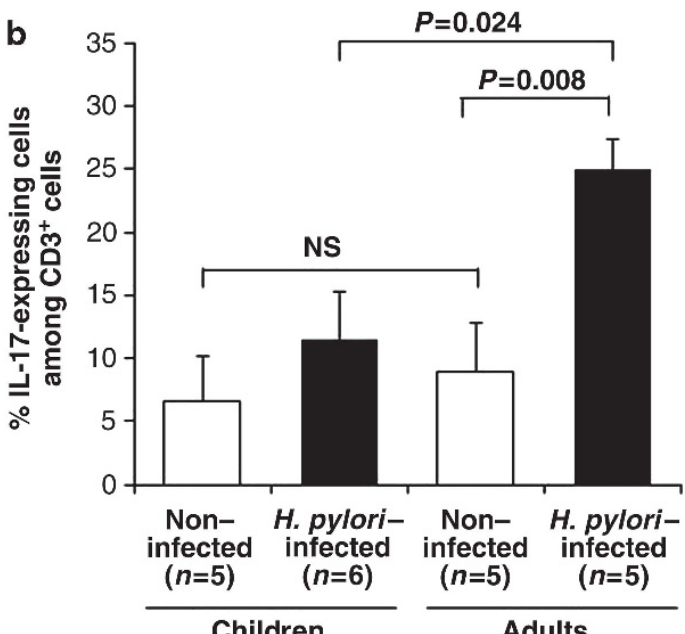

Figure 3 Interleukin (IL)-17-expressing $\mathrm{CD}^{+} \mathrm{T}$ cells in the gastric mucosa of Helicobacter pylori-infected and uninfected children and adults. (a) Co-localization of $\mathrm{CD}^{+}$and $\mathrm{IL}_{-}-17^{+}$mucosal cells in a gastric tissue section from a representative $H$. pylori-infected adult stained with goat anti-CD3 (Cy3) and goat anti-IL-17 (fluorescein isothiocyanate) antibodies. $\mathrm{Bar}=20 \mu \mathrm{m}$. (b) Percentage (mean \pm s.e.m.) of $\mathrm{CD}^{+}{ }^{+} \mathrm{T}$ cells that co-expressed IL-17 was significantly lower in the $H$. pylori-infected children $(n=6)$ compared with that of the infected adults $(n=5)$.

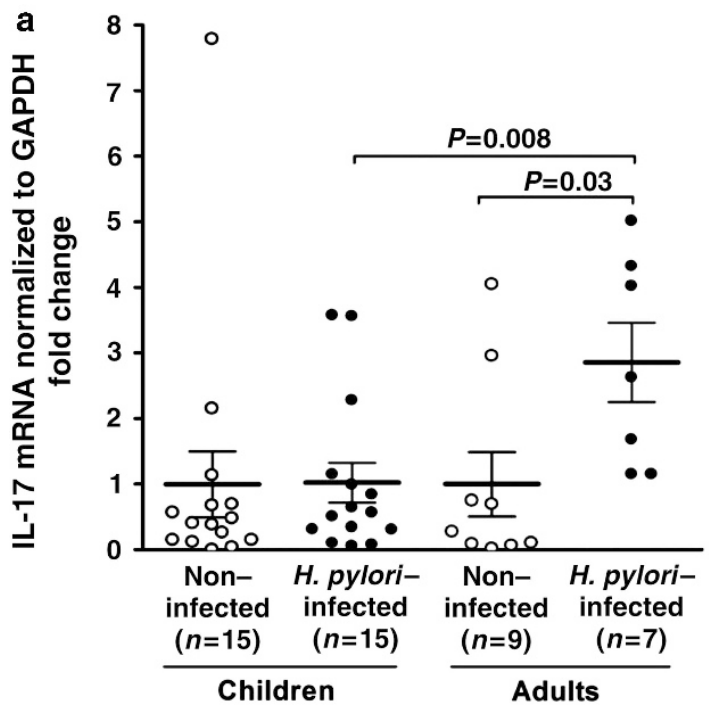

these results, the level of IL-17 mRNA in the children did not correlate with the magnitude of $H$. pylori colonization $(r=-0.09)$ compared with the moderate positive correlation between the levels of IL-17 mRNA and colonization in the adults $(r=+0.3)$. Consequently, the expression of IL-17 mRNA in the mucosa of $H$. pyloriinfected children was significantly lower than that of the infected adults $(P=0.008$; Figure $4 a)$. Among the subjects whose gastric tissue specimens provided sufficient total protein to analyze for IL-17 protein, gastric tissue from the H. pyloriinfected adults, but not the infected children, had higher levels of IL-17 protein compared with that of the uninfected subjects ( $P=0.04$; Figure $4 \mathbf{b}$ ), and, consistent with these findings, the level of IL-17 protein in the infected children was significantly reduced compared with that of the infected adults $(P=0.02)$. The level of IFN- $\gamma$ mRNA in the gastric tissue of infected children was also significantly lower than that of similarly infected adults (data not shown; $P=0.02$ ), corroborating our earlier finding of a reduced Th1 response in the H. pyloriinfected children. ${ }^{8}$ Thus, the gastric IL-17 response, similar to the IFN- $\gamma$ response, was significantly lower in the $H$. pyloriinfected children compared with the response of infected adults.

\section{Mucosal neutrophil infiltration correlates with IL-17 mRNA in the $\boldsymbol{H}$. pylori-infected adults but not children}

As polymorphonuclear neutrophil accumulation is a central feature of $H$. pylori gastritis and can be induced by IL-17, we investigated the relationship between tissue IL-17 responses and neutrophil accumulation in the gastric mucosa of H. pylori-infected children and adults. Among the 15 children with $H$. pylori infection, only 3 of 15 (20\%) had high levels of neutrophil infiltration (neutrophil score 2-3) into the gastric mucosa, whereas, among the 7 adults with infection, 4 (57\%) had similar amounts of high neutrophil infiltration (Figure 5).

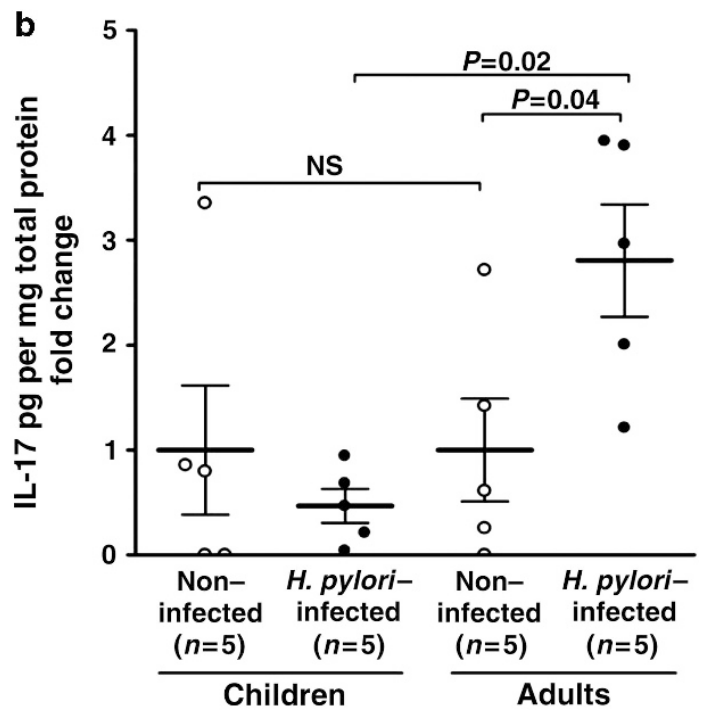

Figure 4 Interleukin (IL)-17-specific mRNA and protein in the gastric mucosa of non-infected and Helicobacter pylori-infected children and adults. IL-17 (a) mRNA and (b) protein in the H. pylori-infected children were significantly lower compared with that of the infected adults. mRNA expression was determined by real-time PCR and normalized to the housekeeping gene GAPDH (glyceraldehyde 3-phosphate dehydrogenase). IL-17 pg protein was determined by enzyme-linked immunosorbent assay and normalized to total $\mathrm{mg}$ protein. Data are presented as fold change ( \pm s.e.m.) in the H. pylori-infected children and infected adults compared with the non-infected cohort subjects. NS, not significant. 


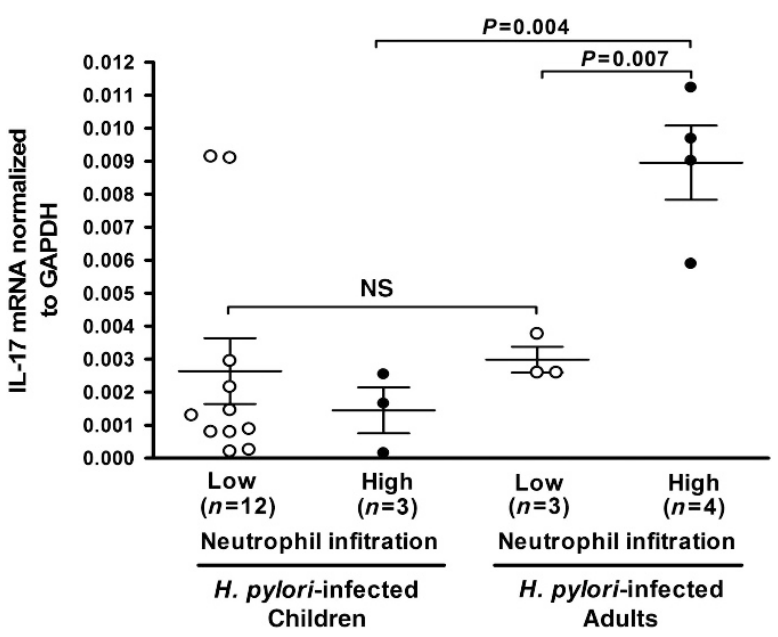

Figure 5 Levels of gastric interleukin (IL)-17 mRNA and polymorphonuclear neutrophil infiltration in the Helicobacter pyloriinfected children and adults. Gastric mucosa from $H$. pylori-infected children $(n=15)$ and adults $(n=7)$ were analyzed for neutrophil infiltration and IL-17 mRNA expression as described in the Methods. Low neutrophil infiltration $=$ neutrophil score $0-1$ and high neutrophil infiltration $=$ neutrophil score 2-3. Gastric IL-17 mRNA responses were lower in the H. pylori-infected children than in the infected adults. Values are presented as mean IL-17 mRNA normalized to GAPDH (glyceraldehyde 3-phosphate dehydrogenase) mRNA \pm s.e.m. NS, not significant.

Moreover, among the infected children with high neutrophil infiltration, the level of IL-17 mRNA was significantly reduced compared with that of the infected adults with the same amount of neutrophil infiltration $(P=0.004)$. Thus, in the gastritis of H. pylori-infected children (Figure 1a), the decrease in neutrophil accumulation was associated with a significantly reduced mucosal IL-17 response.

\section{Increased levels of IL-10 and Foxp3 mRNA in the gastric mucosa of $\boldsymbol{H}$. pylori-infected children compared with the infected adults}

To elucidate the mechanism by which the Th17 response might be downregulated in children infected with $H$. pylori, we analyzed the same gastric tissue specimens for evidence of Treg cell responses. As shown in Figure 6a, gastric H. pylori infection in children was associated with a significant increase in the mucosal level of mRNA for the downregulatory cytokine IL-10 compared with that of the non-infected children $(P=0.03)$. By contrast, $H$. pylori infection in adults was not associated with increased levels of IL-10 mRNA. Importantly, the gastric mucosa of $\mathrm{H}$. pylori-infected children contained significantly higher levels of IL-10 mRNA compared with that of the infected adults $(P=0.03)$. Consistent with the increased gastric IL-10 response in the $H$. pylori-infected children, IL- $10^{+}$cells were identified throughout the lamina propria, albeit at low frequency (Figure 6b). IL-10 ${ }^{+}$cells were also identified in the deep regions of the gastric glands, but more frequently than in the lamina propria, of infected children (Figure 6b). The cells that expressed IL-10 in the lamina propria were exclusively $\mathrm{CD}^{+}{ }^{+} \mathrm{T}$ cells and not HLA-DR ${ }^{+}$cells, whereas the IL-10- expressing cells in the glands were either $\mathrm{CD}^{+}{ }^{+} \mathrm{T}$ cells or cytokeratin $^{+}$epithelial cells (Figure 6b).

The gastric mucosa of $H$. pylori-infected children also had higher levels of mRNA for the Treg transcription factor Foxp3 than that of both the non-infected children and infected adults (Figure 6c), although the increase did not reach statistical significance. In addition, the Foxp3 and IL-17 mRNA levels in the infected children exhibited a negative correlation $(r=-0.08)$ compared with the positive, albeit not significant, correlation in adults $(r=+0.25)$. Together, the increased IL-10 response and prominent Foxp3 response are consistent with an enhanced Treg response in the gastric mucosa of $H$. pylori-infected children.

\section{DISCUSSION}

To elucidate the mucosal regulation of $H$. pylori infection in children, which provides a window into the early host response to the bacteria, we characterized the bacteria, associated cellular infiltrate, and mucosal cytokine response to the infection in children (and adults) residing in Santiago, Chile. Using pediatric and adult cohorts completely different from our previous study, ${ }^{8}$ we first confirmed that the $H$. pylori-infected children have reduced gastric inflammation compared with the infected adults, despite similar mean levels of $H$. pylori colonization. Importantly, inflammation in the children was reduced at each level of bacterial colonization compared with that of adults, suggesting an overall downregulation of the immune-mediated response to $H$. pylori in children. In addition, sequence analysis showed that the bacteria isolated from the infected children and adults had similar cagA and $v a c A$ ( $11 b$ and $\mathrm{s} 2$ ) gene profiles and, in the first genotype analysis of $H$. pylori in Chile, that the bacteria were exclusively of European phylogeographic origin. Although genetic changes due to homologous sequence recombination commonly occur in $H$. pylori over time, ${ }^{23}$ our gene expression studies indicate that differences in common virulence factors and bacterial strains are not the cause of the reduced inflammation in the H. pylori-infected children compared with the infected adults residing in this highly endemic region of the world.

The presence of only hpEurope strains in our subjects is consistent with the European ancestry in approximately twothirds of the Chilean population, especially that of the cities. ${ }^{24}$ Amerindians or persons of Amerindian admixture constitute most of the remaining third and persons of African descent $<0.1 \%$ of the population. ${ }^{25}$ In other regions of South America, persons of African ethnicity represent a substantial proportion of the population. In Colombia, for example, persons with at least some African ancestry represent $21 \%$ of the population and are concentrated along the Caribbean and Pacific coasts. ${ }^{26}$ In the Pacific coast region, up to two-thirds of the inhabitants were recently shown to be colonized by hpAfrical strains, ${ }^{27}$ likely introduced there in the nineteenth century through the importation of slaves infected with African strains. ${ }^{20,21}$ Importantly, strains of African origin dominated in the coastal residents and were less strongly associated with gastric cancer than European strains in the mountain residents. ${ }^{27}$ By contrast, 


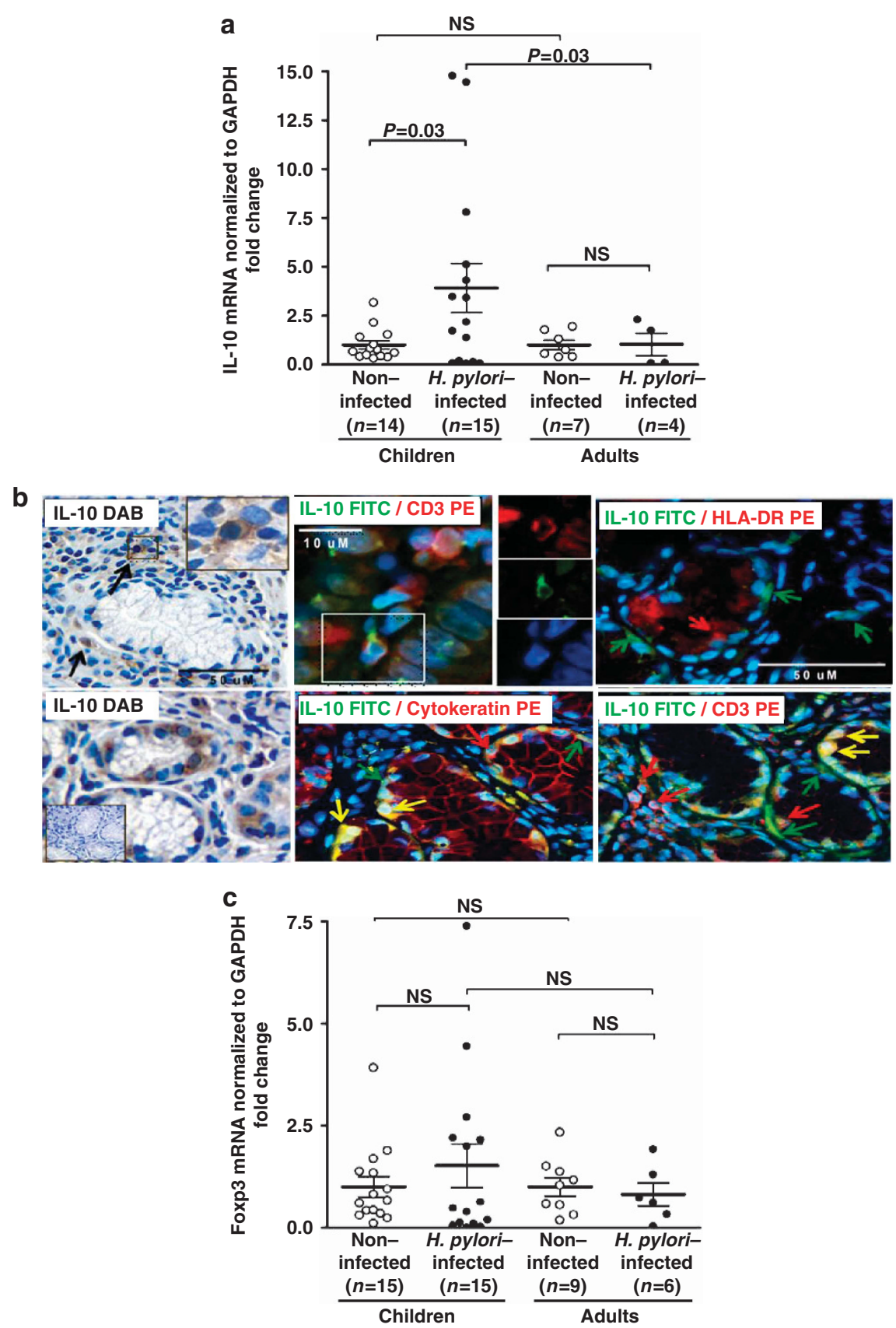

Figure 6 Interleukin (IL)-10 and Foxp3 responses in the gastric mucosa of non-infected and Helicobacter pylori-infected children and adults. Gastric tissue specimens were analyzed for (a) IL-10 mRNA, (b) identity of the IL-10-expressing cells, and (c) Foxp3 mRNA. Levels of IL-10 and Foxp3 mRNA, determined by real-time PCR and normalized to GAPDH (glyceraldehyde 3-phosphate dehydrogenase) mRNA, were higher in the H. pylori-infected children than in the infected adults. Gastric IL-10 protein, detected by immunohistochemistry (left upper and lower panels) and immunofluorescence (right panels) in an $\mathrm{H}$. pylori-infected child, co-localized in the lamina propria in $\mathrm{CD}^{+}{ }^{+}$cells and in the epithelium in cytokeratin ${ }^{+}$ epithelial cells and $\mathrm{CD}^{+} \mathrm{T}$ cells. Inset in the left lower panel shows control tissue without DAB (diaminobenzidine) staining. In the immunofluorescence panels, red and green arrows point to cells that stain with the indicated antibody of the same color; yellow arrows point to the merge, indicating co-localization. FITC, fluorescein isothiocyanate; NS, not significant; PE, phycoerythrin.

the high prevalence of only hpEurope strains in the Chilean children reported here assures prolonged exposure to hpEurope strains as the children develop into infected adults that together with the high frequency of cancer-associated cagA ${ }^{+}$ and $v a c A-s 1^{+}$genotypes ${ }^{28,29}$ likely contributes to the high rate of gastric cancer in the Chilean population.
IL-17 is the signature cytokine produced by Th17 cells and has a mediator role in the inflammation associated with certain autoimmune diseases and host defense against bacterial and fungal pathogens, particularly at mucosal surfaces. ${ }^{30-32}$ Therefore, we characterized the mucosal Th17/IL-17 response in children and adults with $H$. pylori-associated gastritis. Whereas 
no differences in the frequency of Th17 cells were detected in the gastric mucosa of children and adults without $H$. pylori infection, children infected with the bacteria had significantly fewer gastric Th17 cells and significantly lower levels of gastric IL-17-specific mRNA and protein compared with that of similarly infected adults, indicating a potent reduction in the mucosal Th17 response in the children. The gastric mucosa of the infected children also contained less IFN- $\gamma$ mRNA, corroborating our earlier finding of a reduced Th1 response in the H. pylori-infected children. ${ }^{8}$ These findings indicate that adult $H$. pylori gastritis is the consequence of both Th17 and Th1 immune-mediated inflammatory pathways and that both pathways are downregulated in the gastric mucosa of infected children.

Within the gastric inflammatory lesion of $H$. pylori-infected children, neutrophil infiltration was significantly lower and was associated with a reduced IL-17 response compared with that of the infected adults. Thus, IL-17-producing cells in the H. pylori-infected gastric mucosa likely contribute to local neutrophil accumulation, but this contribution is significantly less in children. H. pylori infection in the children was also accompanied by higher mucosal levels of mRNA for the transcription factor Foxp3 and the downregulatory cytokine IL-10, consistent with an enhanced Treg response that may downregulate the Th17-mediated gastric inflammation in the children. The increased IL-10 response is consistent with the finding of higher numbers of IL- $10^{+}$cells among $\mathrm{CD} 3^{+} \mathrm{T}$ cells in the lamina propria and epithelium of $\mathrm{H}$. pylori-infected children. In addition to the presence of IL- $10^{+} \mathrm{CD}^{+} \mathrm{T}$ cells in the epithelium, we also detected IL- $10^{+}$cytokeratin ${ }^{+}$cells in the epithelium, consistent with an epithelial source of IL-10 that has been reported by others. ${ }^{33}$ In mice, the reciprocal polarization between Treg and Th17 cells is associated with inflammation of the central nervous system ${ }^{34,35}$ and colon ${ }^{36}$ and commensal microbiota in the small intestine. ${ }^{37}$ In the mouse stomach, Kao et al. ${ }^{18}$ and Zhang et al. ${ }^{38}$ showed that H. pylori-induced dendritic cells skew the Th17/Treg balance toward a Treg-biased response that suppresses Th17 immunity through a CagA- and VacA-independent, TGF- $\beta$ (transforming growth factor- $\beta$ )-, and IL-10-dependent mechanism. In support of these findings, we recently showed that $H$. pylori was capable of stimulating human gastric dendritic cells to produce IL-10, ${ }^{39,40}$ potentially supplementing Treg suppression of inflammation in the gastric mucosa. Although we have shown that macrophages in normal human intestinal mucosa are incapable of TGF- $\beta$ and IL-10 production, ${ }^{41,42}$ the ability of human gastric macrophages to produce downregulatory cytokines is not known.

Our finding that the $H$. pylori-infected children have downregulated Th17 responses in the gastric mucosa has important implications for the development of an effective $H$. pylori vaccine. First, in animal model studies, IL-17 has been implicated as a key mediator of vaccine-induced reduction in H. pylori infection ${ }^{43-45}$ through the stimulation of local chemokines, including MIP-2 (macrophage-inflammatory protein-2), KC (keratinocyte chemokine), and LIX (lipopolysaccharide-induced CXC chemokine), which recruit neutrophils to the gastric mucosa. ${ }^{43}$ Second, as children will be an especially important target population for $\mathrm{H}$. pylori-specific vaccination in resource-poor nations, reduction in the gastric IL-17 response in children infected with $H$. pylori is a potentially confounding issue that will need to be addressed in the development of such a vaccine. Thus, the Th17/IL-17 results presented here implicate discordant regulation of the gastric mucosal response of children and adults to $H$. pylori. Further elucidation of the immune-mediated mechanism(s) of H. pylori-induced mucosal inflammation during early host development will provide important information for the design of more effective intervention strategies against this challenging pathogen, especially for children in countries with high rates of $H$. pylori infection and gastric cancer.

\section{METHODS}

Patients. Consecutive subjects with abdominal symptoms residing in Santiago, Chile, including 30 children $<13$ years of age and 16 adults $>18$ years of age, were evaluated in this IRB (Institutional Review Board)-approved study. The mean age of the children was $10.4 \pm 2.9$ years old and the adults $48 \pm 13.1$ years old. Female gender dominated in both the children (62\%) and the adults (69\%). All subjects were of European ethnicity, and the mean socioeconomic levels based on the Department of Economy criteria were similar for the groups. Acceptance into the study was based on the presence of symptoms of recurrent abdominal pain, burning abdominal discomfort, hematemesis, or chronic vomiting. Exclusion criteria included (a) use of antibiotics, antacid, $\mathrm{H}_{2}$-blocker, proton-pump inhibitor, bismuth compound, non-steroidal anti-inflammatory drug, or immunosuppressive agent during the 2 weeks before endoscopy; (b) presence of an autoimmune disease; and (c) stool exam positive for ova or parasites.

Assessment of $\boldsymbol{H}$. pylori infection. Subjects provided six antral biopsies by esophagogastroduodenoscopy for the following studies: (1) rapid urease test (Rapid Urea Test; Bios Chile, Santiago, Chile) to detect $H$. pylori; (2) histological analysis and microscopic detection of H. pylori; (3) genotype analysis; (4) immunofluorescence studies; (5) cytokine protein determination; and (6) analysis of cytokine gene expression. A study subject was judged colonized with $H$. pylori if either the rapid urease test or the microscopic evaluation was positive for the bacteria.

Evaluation of gastric $\boldsymbol{H}$. pylori histopathology and colonization. Serial sections of formalin-fixed, paraffin-embedded, hematoxylin and eosin-stained gastric tissue specimens were evaluated for inflammation and bacterial colonization by two pathologists without knowledge of other results. Briefly, five random fields in three tissue sections per biopsy were scored (0-3) according to the modified Sydney classification system ${ }^{46}$ for the level of neutrophils and mononuclear cells, number of lymphoid follicles, mucosal atrophy, and intestinal metaplasia. Thereafter, a histology score reflecting the level of inflammation ${ }^{8}$ was determined by adding the score of each parameter. Similarly, a neutrophil score reflected the level of neutrophils in five random fields in three sections per coded biopsy with $0=$ absent, $1=$ mild, $2=$ moderate, and $3=$ high . A colonization score was calculated based on $H$. pylori density $(0=$ absent, $1=$ mild, $2=$ moderate, and $3=$ intense).

$\boldsymbol{H}$. pylori virulence factor and genotype analysis. Gastric biopsies were placed in Brucella broth, frozen immediately in liquid nitrogen, and subsequently cultured on plates with Brucella agar, 5\% horse blood, and $H$. pylori-selective supplement (Dent; Oxoid, Basingstoke, 
UK). The plates were cultured for 7 days in $10 \% \mathrm{CO}_{2}$ and then passaged three times. A single colony was identified as $H$. pylori using colony morphology and the rapid urease test (Rapid Urea Test, Bios Chile). DNA from the remaining colonies was extracted using the Qiagen DNeasy Minikit (Qiagen, Valencia, CA) and quantified by spectrophotometry. Virulence factor cagA was amplified by PCR, as previously described. ${ }^{47}$ Strains without cagA amplification by PCR were confirmed as cagA negative by amplifying the cag pathogenicity island empty site as previously described. ${ }^{48}$ To analyze the s-region of $v a c A$, the nucleotide sequences of PCR products were compared with variants of four known vacA s-region subtypes (s1a, s1b, s1c, and s2). ${ }^{49}$

Nucleotide sequences for $H$. pylori housekeeping genes (ureI, mut $Y$, efp, $p p a$, atpA, and $\operatorname{trpC}$ ) were also amplified by PCR. ${ }^{20,50}$ PCR products were subsequently purified using the Wizard SV gel and PCR Clean-up kit (Promega, Fitchburg, WI) and stored at $-20^{\circ} \mathrm{C}$. PCR products were sequenced by the University of Alabama at Birmingham DNA Sequencing and Analysis Core. To determine the relationship between strains of $H$. pylori-infecting patients in this study and globally distributed $H$. pylori isolates, we retrieved data from a multi-locus sequence typing (MLST) database (http://pubmlst.org/ helicobacter) for 331 reference isolates with appropriate geographical distribution. Concatenated nucleotide sequences for $H$. pylori MLST loci (ureI, mutY, efp, $p p a$, atpA, and $\operatorname{trp} C$ ) from seven isolates obtained from children and three isolates obtained from adults as well as the 331 isolates retrieved from the MLST database were analyzed using Mega4 software (The Biodesign Institute, Tempe, AZ). ${ }^{51}$ Sequences were aligned using the ClustalW algorithm and phylogenetic relationships were produced using the Kimura two-parameter model for nucleotide substitution and neighbor-joining clustering. A phylogenetic structure was constructed as a bootstrap consensus tree inferred from 1,000 replicates.

Identification and enumeration of gastric Th17 cells. Snap-frozen biopsies were cut into $5 \mu \mathrm{m}$ sections, fixed in acetone $(10 \mathrm{~min})$, and rinsed in phosphate-buffered saline with $0.05 \%$ Tween. After blocking in Dako serum-free protein block for $30 \mathrm{~min}$ at room temperature, the sections were incubated with goat anti-human IL-17 $\left(10 \mu \mathrm{g} \mathrm{ml}^{-1}\right.$; R\&D Systems, Minneapolis, MN) and rabbit anti-human CD3 (1:300; Sigma-Aldrich, St Louis, MO) for $4 \mathrm{~h}$ at room temperature, followed by incubation with Cy3 donkey anti-goat immunoglobulin G (IgG; 1:200; Jackson Immunity, West Grove, PA) and fluorescein isothiocyanate donkey anti-rabbit IgG (1:50; Jackson Immunity) for $30 \mathrm{~min}$ at room temperature. Isotype-matched, irrelevant antibodies were included as controls with each staining experiment. Cell nuclei were labeled with DAPI (4,6-diamidino-2-phenylindole). Sections were mounted, sealed, and stored at $4{ }^{\circ} \mathrm{C}$ until analysis by fluorescence microscopy (Nikon Eclipse T2000-U (Nikon Instruments Inc., Melville, NY), equipped with a CoolSnap ES digital camera and NIS Elements BR2.30 software). The proportion of $\mathrm{IL}_{-1} 7^{+}$cells in the $\mathrm{CD}^{+}$T-cell population was determined on digital images by counting $\mathrm{CD} 3{ }^{+}$cells with and without co-expression of IL-17 using ImageJ1.46R software (Wayne Rasband, National Institutes of Health, USA, hppt://imagej.nih.gov/ij). Regions of interest were set to exclude surface and glandular epithelial cells. Three or more randomly selected areas from two slides per specimen were analyzed by two independent investigators.

Identification of gastric IL-10 cells. Biopsies fixed in $4 \%$ formalin, embedded in paraffin, and cut in $7 \mu \mathrm{m}$ sections were rehydrated and rinsed in water. Sections were subjected to antigen retrieval (citrate buffer, DAKO Target Retrieval Solution, DAKO, Carpinteria, CA; $20 \mathrm{~min}>92^{\circ} \mathrm{C}$ by microwave), endogenous peroxidase inhibition, and casein protein blocking (each $20 \mathrm{~min}$, room temperature, CSA System DAKO), and incubated overnight at $4{ }^{\circ} \mathrm{C}$ with primary antibody (DAKO Antibody Diluent with Background Reducing Components). Primary antibodies included (a) mouse anti-human IL-10 IgG2b (1:200, Santa Cruz Biotechnology, Santa Cruz, CA); (b) rabbit anti-human CD3 IgG (1:400, Sigma-Aldrich); (c) mouse anti-human HLA-DR IgG1 (1:20, BD Biosciences, San Jose, CA); and (d) mouse anti-human cytokeratin IgG1 (1:50, Cell Signaling, Danvers, MA). IL-10-positive cells were identified by tyramide signal amplification (1:100, goat anti-mouse IgG2b-horseradish peroxidase; 1:50, biotinylated tyramide; 1:1,000, streptavidin-horseradish peroxidase, PerkinElmer, Shelton, CT) followed by diaminobenzidine staining and counterstained with hematoxylin or by tyramide signal amplification as above using tyramide-fluorescein isothiocyanate (1:50, Molecular Probes 488, Eugene, OR). Nuclei were counterstained with DAPI (1:500). $\mathrm{CD}^{+}$cells were detected with donkey anti-rabbit IgG (1:400, Jackson Immunity); HLA-DR ${ }^{+}$and cytokeratin ${ }^{+}$cells were detected with goat anti-mouse IgG1 (1:100, Molecular Probes 594) and nuclei were counterstained with DAPI. Control slides were included with every experiment $(n=12)$. Sections were stored and analyzed as above.

Cytokine protein determination. Biopsy specimens were homogenized in $750 \mu \mathrm{l}$ phosphate-buffered saline, centrifuged $(12,000 \mathrm{~g}$, $\left.5 \mathrm{~min}, 4^{\circ} \mathrm{C}\right)$, and the supernatant collected and frozen $\left(-70^{\circ} \mathrm{C}\right)$ until assayed. The concentration of IL-17 was measured by enzyme-linked immunosorbent assay (R\&D Systems, Minneapolis, MN) and expressed as $\mathrm{pg} \mathrm{mg}^{-1}$ protein. Total protein was measured using the bicinchoninic acid method (Pierce, Rockford, IL) and expressed as $\mathrm{mg} \mathrm{ml}^{-1}$

Real-time reverse transcriptase-PCR analysis for cytokine gene expression. Gastric specimens were snap frozen in liquid nitrogen and kept at $-80^{\circ} \mathrm{C}$ until processed. Total RNA was extracted using the Qiagen RNeasy Minikit (Qiagen). RNA concentration and purity were determined by spectrophotometry, and RNA integrity was assesed by agarose gel electrophoresis. RNA then was treated with DNAse I (Invitrogen, Carlsbad, CA) for $15 \mathrm{~min}$, heat inactivated at $65^{\circ} \mathrm{C}$ to avoid genomic DNA amplification during real-time PCR and reverse transcribed into first-strand cDNA using the affinityScript qPCR cDNA synthesis kit (Agilent Technologies, Santa Clara, CA) with oligo $\mathrm{dT}$ as primers. Real-time PCR was performed on duplicate cDNA samples with custom-made primer sets using Brilliant II SYBR green QPCR master mix in an Mx3000p Real-Time PCR System machine (both from Agilent Technologies). The following primer pairs for IL-17 (FW: 5' -ACCAATCCCAAAAGGTCCTC-3'; RV: 5' -GGGGAC AGAGTTCATGTGGT-3'); IL-10 (FW: $5^{\prime}$-GTGATGCCCCAAGCT GAGA-3'; RV: 5' -CACGGCCTTGCTCTTGTTTT-3'); Foxp3 (FW: 5'-AGAAGCAGCGGACACTCAAT-3'; RV: 5'-GAAAGGAGGA TGGACGAACA-3'); IFN- $\gamma$ (FW: $5^{\prime}$-GAATTGGAAAGAGGAGAG TGAC-3'; RV: 5'-TGTATTGCTTTGCGTTGGAC-3'); and GAPDH (glyceraldehyde 3-phosphate dehydrogenase; reference gene) (FW: $5^{\prime}$-AACCTGCCAAATATGATGAC- ${ }^{\prime}$; RV: $5^{\prime}$-GTTGTCATACCA GGAAATGAG-3') were used for amplification.

PCR fragments for each cytokine and the reference gene obtained by reverse transcriptase-PCR were cloned into the pGEM-T Easy Vector System (Promega). Plasmids extracted from selected clones using the Wizard SV miniprep DNA purification systems (Promega) containing expected inserts were quantified by spectrophotometry. Serial dilutions of the vector insert were used as standard curves to quantify the levels of gene expression. mRNA levels were analyzed by comparing the differences in fold change in cytokine mRNA normalized to GAPDH mRNA.

Statistical analysis. Comparisons between groups were performed using the Student's $t$-test or the Mann-Whitney $U$ test when appropriate for continuous data. Categorical data were analyzed using the Chi-square test and the Fisher's exact test. Spearman's rank correlation and the Goodness-of-Fit test were used to analyze relationships, although only the Spearman correlation coefficient is presented as analyses were uniformly consistent with each other. Comparison of curve fit values was performed using an F-test. Statistical significance was defined as a $P$ value of $<0.05$. 


\section{ACKNOWLEDGEMENTS}

We thank Robin G. Lorenz, M.D., Ph.D. for assistance with the tyramide signal amplification. This study was supported by Fondecyt (\#1100654, \#1085232) and Conicyt RUE29 (Chile); the National Institutes of Health (DK-54495, DK-84063, Al-83539, Al-83027, RR-20136, Al068009, CA116087, and the Cell and Molecular Pathology Core of the UAB Mucosal HIV and Immunobiology Center, DK-64400); UAB Autoimmunity, Immunology and Transplantation Pilot Program; UAB Center for Clinical and Translational Science Pilot Program (UL1, TR000165); the Department of Veterans Affairs; and the Fulbright Fellowship Program, Department of State (USA).

\section{DISCLOSURE}

The authors declared no conflict of interest.

c 2013 Society for Mucosal Immunology

\section{REFERENCES}

1. Mohammadi, M., Czinn, S., Redline, R. \& Nedrud, J. Helicobacter-specific cell-mediated immune responses display a predominant Th1 phenotye and promote a delayed-type hypersensitivity response in the stomachs of mice. J. Immunol. 156, 4729-4738 (1996).

2. Smythies, L.E., Waites, K.B., Lindsey, R.J., Harris, P.R., Ghiara, P. \& Smith, P.D. Helicobacter pylori-induced mucosal inflammation is Th1 mediated and exacerbated in IL-4, but not IFN- $\gamma$, gene-deficient mice. J. Immunol. 165, 1022-1029 (2000).

3. Mattapallil, J.J., Dandekar, S., Canfield, D.R. \& Solnick, J.V. A predominant Th1 type of immune response is induced early during acute Helicobacter pylori infection in rhesus macaques. Gastroenterology 118, 307-315 (2000).

4. Karttunen, R., Karttunen, T., Ekre, H.-P.T. \& MacDonald, T.T. Interferon gamma and interleukin 4 secreting cells in the gastric antrum in Helicobacter pylori positive and negative gastritis. Gut 36, 341-345 (1995).

5. Haeberle, H.A. et al. Differential stimulation of interleukin-12 (IL-12) and IL10 by live and killed Helicobacter pylori in vitro and association of IL-12 production with $\gamma$ interferon-producing $T$ cells in the human gastric mucosa. Infect. Immun. 65, 4229-4235 (1997).

6. D'Elios, M.M. etal. Thelper 1 effector cells specific for Helicobacter pylori in the gastric antrum of patients with peptic ulcer disease. J. Immunol. 158, 962-967 (1997).

7. Bamford, K.B. et al. Lymphocytes in the human gastric mucosa during Helicobacter pylori have a Thelper cell 1 phenotype. Gastroenterology 114 482-492 (1998).

8. Harris, P.R. et al. Helicobacter pylori gastritis in children is associated with a regulatory T-cell response. Gastroenterology 134, 491-499 (2008).

9. Guiraldes, E. et al. Proinflammatory cytokine expression in gastric tissue from children with Helicobacter pylori-associated gastritis. J. Pediatr. Gastroenterol. Nutr. 33, 127-132 (2001).

10. Whitney, A.E., Guarner, J., Hutwagner, L. \& Gold, B.D. Helicobacter pylori gastritis in children and adults: comparative histopathologic study. Ann. Diagn. Pathol. 4, 279-285 (2000).

11. Lundgren, A. et al. Mucosal FOXP3-expressing CD4 ${ }^{+}$CD25high regulatory T cells in Helicobacter pylori-infected patients. Infect. Immun. 73, 523-531 (2005).

12. Robinson, K. et al. Helicobacter pylori-induced peptic ulcer disease is associated with inadequate regulatory $\mathrm{T}$ cell responses. Gut $\mathbf{5 7}$, 1375-1385 (2008).

13. Raghavan, S., Fredriksson, M., Svennerholm, A.M., Holmgren, J. \& SuriPayer, E. Absence of $\mathrm{CD} 4{ }^{+} \mathrm{CD} 25^{+}$regulatory T cells is associated with a loss of regulation leading to increased pathology in Helicobacter pyloriinfected mice. Clin. Exp. Immunol. 132, 393-400 (2003).

14. Rad, R. et al. CD25 $5^{+} / \mathrm{Foxp}^{+} \mathrm{T}$ cells regulate gastric inflammation and Helicobacter pylori colonization in vivo. Gastroenterology 131, 525-537 (2006).

15. Alam, M.S. et al. CD73 is expressed by human regulatory Thelper cells and suppresses proinflammatory cytokine production and Helicobacter felisinduced gastritis in mice. J. Infect. Dis. 199, 494-504 (2009).
16. Shiomi, S. et al. IL-17 is involved in Helicobacter pylori-induced gastric inflammatory responses in a mouse model. Helicobacter 13, 518-524 (2008).

17. Algood, H.M., Gallo-Romero, J., Wilson, K.T., Peek, R.M. Jr \& Cover, T.L. Host response to Helicobacter pylori infection before initiation of the adaptive immune response. FEMS Immunol. Med. Microbiol. 51, 577-586 (2007).

18. Kao, J.Y. et al. Helicobacter pylori immune escape is mediated by dendritic cell-induced Treg skewing and Th17 suppression in mice. Gastroenterology 138, 1046-1054 (2010).

19. Fox, J.G. \& Wang, T.C. Inflammation, atrophy, and gastric cancer. J. Clin. Invest. 117, 60-69 (2007).

20. Falush, D. et al. Traces of human migrations in Helicobacter pylori populations. Science 299, 1582-1585 (2003).

21. Linz, B. et al. An African origin for the intimate association between humans and Helicobacter pylori. Nature 445, 915-918 (2007).

22. Caruso, R. et al. IL-23-mediated regulation of IL-17 production in Helicobacter pylori-infected gastric mucosa. Eur. J. Immunol. 38, 470-478 (2008).

23. Kraft, C. et al. Genomic changes during chronic Helicobacter pylori infection. J. Bacteriol. 188, 249-254 (2006).

24. Cruz-Coke, R. \& Moreno, R.S. Genetic epidemiology of single gene defects in Chile. J. Med. Genet. 31, 702-706 (1994).

25. Medina, E.L. \& Kaempffer, A.M.R. Elementos de Salud Publica. Biblioteca Digital. Universidad de Chile2011). Section 5.2.6.

26. National Administration Department of Statistics (DANE). Government of Columbia (2007).

27. de Sablet, T. et al. Phylogeographic origin of Helicobacter pylori is a determinant of gastric cancer risk. Gut 60, 1189-1195 (2011).

28. Basso, D. et al. Clinical relevance of Helicobacter pylori cagA and vacA gene polymorphisms. Gastroenterology 135, 91-99 (2008).

29. Blaser, M.J. et al. Infection with Helicobacter pylori strains possessing cagA is associated with an increased risk of developing adenocarcinoma of the stomach. Cancer Res. 55, 2111-2115 (1995).

30. Mangan, P.R. et al. Transforming growth factor- $\beta$ induces development of the $T(H) 17$ lineage. Nature 441, 231-234 (2006).

31. Weaver, C.T., Hatton, R.D., Mangan, P.R. \& Harrington, L.E. IL-17 family cytokines and the expanding diversity of effector Tcell lineages. Annu. Rev. Immunol. 25, 821-852 (2007).

32. Ouyang, W., Kolls, J.K. \& Zheng, Y. The biological functions of Thelper 17 cell effector cytokines in inflammation. Immunity 28, 454-467 (2008).

33. Bodger, K., Bromelow, K., Wyatt, J.I. \& Heatley, R.V. Interleukin 10 in Helicobacter pylori associated gastritis: immunohistochemical localisation and in vitro effects on cytokine secretion. J. Clin. Pathol. 54, 285-292 (2001).

34. Bettelli, E. et al. Reciprocal developmental pathways for the generation of pathogenic effector $\mathrm{T}_{\mathrm{H}} 17$ and regulatory T cells. Nature 441, 235-238 (2006).

35. Quintana, F.J. et al. Control of $T_{\text {reg }}$ and $T_{H} 17$ cell differentiation by the aryl hydrocarbon receptor. Nature 453, 65-71 (2008).

36. Mucida, D. et al. Reciprocal $T_{H} 17$ and regulatory $T$ cell differentiation mediated by retinoic acid. Science 317, 256-260 (2007)

37. Ivanov, I.I. et al. Specific microbiota direct the differentiation of IL-17producing T-helper cells in the mucosa of the small intestine. Cell Host Microbe 4, 337-349 (2008).

38. Zhang, M., Liu, M., Luther, J. \& Kao, J.Y. Helicobacter pylori directs tolerogenic programming of dendritic cells. Gut Microbes 1, 325-329 (2010).

39. Bimczok, D. et al. Human primary gastric dendritic cells induce a Th1 response to H. pylori. Mucosal Immunol. 3, 260-269 (2010).

40. Bimczok, D., Grams, J.M., Stahl, R.D., Waites, K.B., Smythies, L.E. \& Smith, P.D. Stromal regulation of human gastric dendritic cells restricts the Th1 response to Helicobacter pylori. Gastroenterology 141, 929-938 (2011).

41. Smythies, L.E. et al. Human intestinal macrophages display profound inflammatory anergy despite avid phagocytic and bacteriocidal activity. J. Clin. Invest. 115, 66-75 (2005).

42. Smythies, L.E. et al. Inflammation anergy in human intestinal macrophages is due to Smad-induced $\mathrm{l \kappa B} \alpha$ expression and NF-kB inactivation. J. Biol. Chem. 285, 19593-19604 (2010). 
43. DeLyria, E.S., Redline, R.W. \& Blanchard, T.G. Vaccination of mice against $H$ pylori induces a strong Th-17 response and immunity that is neutrophil dependent. Gastroenterology 136, 247-256 (2009).

44. Velin, D. et al. Interleukin-17 is a critical mediator of vaccine-induced reduction of Helicobacter infection in the mouse model. Gastroenterology 136, 2237-2246 (2009).

45. Hitzler, I., Oertli, M., Becher, B., Agger, E.M. \& Muller, A. Dendritic cells prevent rather than promote immunity conferred by a helicobacter vaccine using a mycobacterial adjuvant. Gastroenterology 141, 186196 (2011).

46. Dixon, M.F., Genta, R.M., Yardley, J.H. \& Correa, P. Classification and grading of gastritis. The updated Sydney System. International Workshop on the Histopathology of Gastritis, Houston 1994. Am. J. Surg. Pathol. 20, 1161-1181 (1996).
47. van Doorn, L.J. et al. Expanding allelic diversity of Helicobacter pylori vacA. J. Clin. Microbiol. 36, 2597-2603 (1998).

48. Sicinschi, L.A., Correa, P., Bravo, L.E. \& Schneider, B.G. A positive assay for identification of cagA negative strains of Helicobacter pylori. J. Microbiol. Methods 55, 625-633 (2003).

49. Rudi, J. et al. Diversity of Helicobacter pylori vacA and cagA genes and relationship to VacA and CagA protein expression, cytotoxin production, and associated diseases. J. Clin. Microbiol. 36, 944-948 (1998).

50. Achtman, M. et al. Recombination and clonal groupings within Helicobacter pylori from different geographical regions. Mol. Microbiol. 32, 459-470 (1999).

51. Tamura, K., Dudley, J., Nei, M. \& Kumar, S. MEGA4: Molecular Evolutionary Genetics Analysis (MEGA) software version 4.0. Mol. Biol. Evol. 24, 1596-1599 (2007). 